\title{
1 Landscape and dynamics of the transcriptional regulatory network 2 during natural killer cell differentiation
}

$4 \quad$ Kun $\mathrm{Li}^{1,3}$, Yang $\mathrm{Wu}^{2,3}$, Young $\mathrm{Li}^{1}$, Qiaoni $\mathrm{Yu}^{1}$, Zhigang $\operatorname{Tian}^{2}$, Haiming $\mathrm{Wei}^{2}{ }^{2}$, Kun $\mathrm{Qu}^{1, *}$ 5

6 Affiliation

$8{ }^{1}$ Division of Molecular Medicine, Hefei National Laboratory for Physical Sciences at Microscale, 9 the CAS Key Laboratory of Innate Immunity and Chronic Disease, CAS Center for Excellence in 10 Molecular Cell Science, School of Life Sciences, University of Science and Technology of China.

$11{ }^{2}$ Division of Molecular Medicine, Hefei National Laboratory for Physical Sciences at Microscale,

12 the CAS Key Laboratory of Innate Immunity and Chronic Disease, School of Life Sciences, 13 University of Science and Technology of China.

$14{ }^{3}$ Co-first authors

*Correspondence: Kun Qu (qukun@ustc.edu.cn) and Haiming Wei (ustcwhm@ustc.edu.cn)

\section{Contact Information}

Kun Qu Ph.D.

21 Division of Molecular Medicine, Hefei National Laboratory for Physical Sciences at Microscale,

22 the CAS Key Laboratory of Innate Immunity and Chronic Disease, CAS Center for Excellence in

23 Molecular Cell Science, School of Life Sciences, University of Science and Technology of China.

24 Hefei, Anhui, China, 230027

25 Email: qukun@ustc.edu.cn

26 Phone: +86-551-63606257 


\section{Abstract}

31 Natural killer (NK) cells are essential in controlling cancer and infection. However, little is known

32 about the dynamics of the transcriptional regulatory machinery during NK cell differentiation. In

33 this study, we applied assay of transposase accessible chromatin with sequencing (ATAC-seq)

34 technique in a self-developed in vitro NK cell differentiation system. Analysis of ATAC-seq data

35 illustrated two distinct transcription factor (TF) clusters that dynamically regulate NK cell

36 differentiation. Moreover, two TFs from the second cluster, FOSL2 and EGR2, were identified as

37 novel essential TFs that control NK cell maturation and function. Knocking down either of these

38 two TFs significantly impacted NK cell transformation. Finally, we constructed a genome-wide

39 transcriptional regulatory network that provides an understanding of the regulatory dynamics

40 during NK cell differentiation.

\section{Keywords}

44 Natural killer; ATAC-seq; programmed differentiation; FOSL2 and EGR2; dynamic regulatory 45 networks

Introduction

Natural killer (NK) cells are innate lymphocytes, and as the name suggests, NK cells

51 during pathogen invasion, which surveys the environment and to protect the host from infection

52 or cancer cells. [1]. Additionally, NK cell-based immunotherapy has become an emerging force in

53 cancer treatment and will play an essential role in the future treatment [2-6]. For instance, adoptive

54 NK cell immunotherapy has become increasingly popular because it induces graft-versus-

55 leukemia effects without causing graft-versus-host disease in patients [7]. Therefore, major efforts

56 are currently underway to fully utilize the anti-tumor properties of NK cells in the clinic. In

57 addition, a variety of methods include the development of NK cell expansion protocols to establish

58 a microenvironment that favors NK cell activity, redirect the activity of NK cells on tumor cells

59 and release the inhibitory signals that limit NK cell function [8]. On the other hand, many 
researchers used umbilical cord blood (UCB) $\mathrm{CD}^{+} 4^{+}$cells to produce abundant NK cells [9-18] that were used for clinical application without feeding cells by adding various cytokines [10]. In our previous work, we have developed a method to obtain sufficient functional NK cells by simply adding a mixture of cytokines and providing a mechanism by which NK cells can be used to treat leukemia [19]. The use of NK cells for immunotherapy relies on a great number of NK cells with optimal cytotoxic activity [4]; therefore, a comprehensive understanding of the regulatory circuits during NK cell differentiation is particularly important for boosting the efficacy of clinical treatments. However, the mechanisms underlying the differentiation of the NK cells are not well understood. Current studies have shown that transcriptional factors (TFs) play an essential role in driving NK cell maturation, and many TFs have been well studied in this process [1]. Additionally, it is known that different TFs play various roles at distinct stages of differentiation [1]. For example, PU.1 is a TF that is known to drive hematopoietic stem cell (HSC) differentiation into the earliest myeloid and lymphoid progenitors [20], whereas T-bet is an essential TF in the control of NK cell maturation and IFN $\gamma$ secretion regulation [21]. However, how TFs work in concert to enforce the NK cell phenotype is not clear. In our work, we investigated the landscape and dynamics of the transcriptional regulatory network during NK cells differentiation from cord blood CD34 ${ }^{+} \mathrm{HSCs}$ based on a newly developed epigenomic profiling technique named ATAC-seq [22].

ATAC-seq has been widely used to profile the epigenetic landscapes of cells at specific stages of interest and thereby delineate the underlying regulatory mechanisms of gene expression. For example, a previous report used ATAC-seq to help identify an exhaustion-specific enhancer that regulates PD-1 expression and thereby elucidated the regulatory mechanism of gene expression in exhausted $\mathrm{CD} 8^{+} \mathrm{T}$ cells $[23,24]$. Alternately, ATAC-seq analysis of pure cancer cell populations of human small cell lung cancer (SCLC) identified a novel TF $N f i b$ which is necessary and sufficient to drive the metastatic ability of SCLC cells [25]. ATAC-seq has also enabled researchers to track the epigenomic states changes in patient-derived immune cells [26], and to survey how the personal regulomes of the cutaneous $\mathrm{T}$ cell lymphoma patients determines their responses towards histone deacetylase inhibitors (HDACi) anti-cancer drugs [27]. More recently,

87 ATAC-seq was applied to better understand the controlling of NK cells in innate immune memory during infection [28], illustrating the importance of the topic as well as the power of the technique used in this study. Here, we have developed a systematic method to characterize the chromatin 
91 ATAC-seq. Motif and enrichment analysis from HOMER [29] and Genomica [26] show that many

92 TFs play important roles in NK cell differentiation, and by integrating gene expression profiles

93 from our previous study [19], we identified two novel transcription factors, FOSL2 and EGR2, that

94 are essential for driving NK cell maturation.

Results

97

Landscape of DNA accessibility during NK cell differentiation

To elucidate the regulatory networks during NK cell differentiation, we developed a culture procedure to obtain differentiated NK cells from UCB CD34 ${ }^{+}$cells using a cocktail of cytokines [19]. The differentiation process requires 35 days. Interestingly, after culturing of cord blood stem cells for 3 weeks, the proportion of NK cells rapidly increased from 5\% on day 21 to approximately $60 \%$ on day 28, and peaking at close to $100 \%$ on day 35 (Figure 1A and Figure S1A) [19]. To elucidate the molecular mechanism and regulatory network underlying NK cell differentiation, we interrogated the landscape of chromatin accessibility using ATAC-seq at 8 different time points, with 2 replicates each along the process (Figure 1B). Multiple bioinformatics analysis methods (see Materials and Methods) were then applied to obtain the differential accessible chromatin sites, enriched transcription factors (TFs) and genome-wide regulatory elements. At least 50,000 cells were obtained from each sample, and on average, resulted more than 78 million reads with a total of 1260 million measurements (Table S1). From this dataset, 143,570 DNA accessible sites were identified $\left(\mathrm{P}<10^{-7}, \mathrm{FDR}<10^{-7}\right)$. Quantitative analysis indicated that the dataset was of high quality with a strong signal to background noise ratio and expected fragment length distribution

114 (Figure S1B-E). Moreover, the dynamics of the chromatin accessibilities around the known surface marker genes were consistent with changes of their gene expressions during NK cell differentiation (Figure S1D) [30, 31]. Pearson correlation analysis on the biological replicates at

117 each time point also showed that our ATAC-seq profiles were highly reproducible (Figure S2AB). Within all the accessible sites, only a very small portion (6.48\%) were conserved across all stages (Figure S3A), while the majority of peaks changed over time, suggesting significant chromatin dynamics during the NK cell differentiation. Peaks that were detected at all stages were 
122 basic physiological activities (Figure S3B). In contrast, stage-specific peaks, especially peaks that 123 emerged at later stage of the process, were highly enriched in immune relevant GO terms, suggesting the activation of critical genes that govern NK cell function.

For instance, several TFs are known to regulate NK cell differentiation, such as SPI1 and TBX21. The former is a NK cell repressor and the latter an activator. Gene expression profiles from our previous study [19] indicated down-regulation of the SPI1 gene and up-regulation of the TBX21 gene during NK cell differentiation (Figure 1C). The protein levels of these two genes obtained from flow cytometric experiments suggested the same trends (Figure 1D). ATAC-seq revealed two regulatory elements at the promoters and intronic enhancers around each of these two genes (Figure 1E). Chromatin-accessible sites around the SPI1 gene clearly became unavailable and those around the $T B X 21$ gene gradually became available during the process, supporting the notion that the epigenetic dynamics of key regulators are consistent with their corresponding gene and protein expressions. The consistency between the epigenetic and gene expression profiles of transcription factors, such as GATA3 and EOMES, which are known to drive NK cell differentiation, are shown in (Figure S1E), indicating the feasibility of predicting transcriptional regulatory networks from ATAC-seq profiles.

\section{Epigenomic signatures of different stages during NK cell differentiation}

To determine the differences in regulatory DNA activity among different stages during NK

142 cell differentiation, we applied pairwise comparisons of the ATAC-seq signals between the corresponding samples, together with intrinsic analysis [32], a method that highlighted elements

144 that varied in accessibility across individuals but not between repeat samples from the same

145 individual. We discovered 6401 peaks of differential DNA accessible sites across the genome, and 146 identified three distinct clusters of chromatin accessible sites via unsupervised hierarchical 147 clustering (Figure 2A, Figure S4A, B). Principal component analysis (PCA) of all the samples 148 also illustrated three distinct clusters, which were consistent with the time process of NK cell 149 differentiation representing the early, intermediate and late stages of the entire process (Figure 150 S4C). Gene ontology analysis of these peaks was performed in GREAT [33]. Cluster I comprises 151 of 1584 elements that were more accessible at the early stage (days7 21) of differentiation. Peaks 152 in cluster I were enriched in the GO terms of metabolic processes, with a certain level of 
153 significance (Figure 2B, $10^{-8}<\mathrm{P}<10^{-4}$ ), keeping cells alive, proliferation and prepare to differentiate.

154 Cluster II comprises 4817 elements, which were highly accessible at the late stage (days 24 35) of NK cell differentiation. GREAT analysis revealed that peaks in cluster II were strongly enriched $\left(\mathrm{P}<10^{-50}\right)$ for immune-relevant GO terms, such as immune system process, immune response and

157 immune system development among others (Figure 2B), indicating that the epigenetic states of functional immune cell-specific genes were activated throughout the process and then drove progenitor cells differentiation toward NK cells. Cluster III consisted of only 356 peaks, which showed no enrichment for GO terms and were therefore neglected in downstream analysis.

We next examined whether the DNA accessibility chromatin signatures at different stages correlated with those of the corresponding gene expressions. By comparing the ATAC-seq profiles with the genome-wide microarray data during NK cell differentiation, we found that gene loci that gained chromatin accessibility were significantly increased in the gene expression level $\left(\mathrm{P}<10^{-6}\right)$, while gene loci that lost chromatin accessibility had decreased expression $\left(\mathrm{P}<10^{-9}\right)$, indicating a high correlation between epigenetic and RNA profiles (Figure 2C).

Chromatin structure and epigenetic modifications regulate gene expression [34], however, phenotype have not yet been well studied. Here, we integrated the ATAC-seq signals, microarray profile and percentage of NK cell counts to examine the temporal dynamics of these three features.

171 Interestingly, we noticed that the accessibility of mature NK cell-specific peaks (cluster II in

172 Figure 2A) started to open up on day 14; and the expression of NK cell specific genes was turned 173 on approximately two days later, while the percentage of NK cell started to grow after day 21

174 (Figure 2D). These results suggested a clear chronological order of the changes in chromatin 175 structure, gene expression and cell phenotype during NK cell differentiation.

\section{Transcription factor occupancy network during NK cell differentiation} seq, and use it to create regulatory networks [26]. To identify potential drivers of NK cell differentiation, we applied HOMER and used both the default (Figure 3A) and setting background (Figure S5A) search modes (see Materials and Methods) to search for TFs that were enriched at 
accessible elements in cluster I and cluster II suggesting that NK cell differentiation and maturation require a variety of transcription factors. TFs enriched at cluster I peaks are potential regulators at the early stage of NK differentiation, while those enriched at cluster II peaks could be critical regulators at the late stage. We found several TF families that were significantly enriched $\left(\mathrm{P}<10^{-}\right.$ ${ }^{10}$ ), and many of which are important known TFs of NK cells differentiation, such as the RUNX and ETS1/PU.1 families [20,35] and CEBP [36], supporting the reliability of this method to detect critical regulators. For instance, PU.1 is widely expressed and controls multiple stages of bone marrow and lymphocyte differentiation in a variety of hematopoietic-derived lineages [37-41], and a reduction in the number of NKPs and iNKs has been detected in chimeric mice [20], indicating that PU.1 may play an important role during the early stages of NK cell differentiation. Several known TFs are also enriched at the late stage, such as RUNX [35], E2A [42], T-bet [21], STAT5 [43], and EOMES [21]. The most enriched motif in cluster II was the RUNX family: RUNX1, RUNX and RUNX2 (Figure S5A), which are key regulators of lymphocyte lineage-specific gene expression [44].

Motif searches of differential ATAC-seq peaks could potentially provide information about the transcriptional regulatory network, but one caveat of this method is unable to distinguish between similar motifs of binding TF family members. Thus, we sought to apply the Genomica's module map algorithm and "TF footprint" analysis to better predict TF occupancy on accessible sites by integrating the ATAC-seq and gene expression microarray profiles (Figure 3).

From the Jasper database we obtained 242 vertebrate TF motifs [45]. We then used Genomica [27] producing a time points-specific TF occupancy network (Figure 3B). This analysis revealed distinct patterns of TF access to DNA at different time points. Many known TFs were enriched, for instance, STAT5, which is an IL-15 downstream signaling molecule and is indispensable throughout the lifetime of NK cells. Deficiency of STAT5a/b has been reported to result in complete elimination of NK cells, which demonstrates the important and non-redundant effects of STAT5 [43]. The JAK-STAT pathway has also been shown to be an important signaling pathway used by various cytokines and growth factors [46]. The interferon regulatory factor (IRF) family regulates a variety of processes, including hematopoietic development, tumorigenesis, host immunization and pathogen defense [47, 48]. IRF2 is required to maintain the normal differentiation of NK cells in a cell-intrinsic manner [49, 50]. And IRF2-deficient NK cells showed reduced levels of mature markers and IFN- $\gamma$ production during stimulation [49, 50]. T-bet and 
EOMES are members of the T-box family and are known to control different aspects of NK cell differentiation and maturation. [21, 51, 52].

In addition, several novel TFs were also enriched, namely, FOSL2 and EGR2, and are potential regulators of NK cell differentiation. These TFs were assessed along with the other enriched TF families from the motif analysis to identify which gene in the family was expressed or differentially expressed during NK differentiation to further filter out true regulators. At each time point, we plotted both the expression value (colored from red to green to represent high to

222 low expression in the gene microarray profile) and the motif enrichment score (shown by the circle 223 size representing the $-\log (\mathrm{P}$-value) of the enrichment) in the same figure (Figure 3C), and we 224 observed that the known regulators were highly expressed and enriched at different stages, same 225 as the genes FOSL2 and EGR2. By integrating results from the above three analyze (Figure 3AC), we predict FOSL2 and EGR2 as potential regulators.

DNA sequences that are directly occupied by DNA-binding proteins are protected from transposition, and therefore the resulting sequence "footprint" reveals the presence of a DNAbinding protein at each site, analogous to DNase digestion footprints. TF "footprint" analysis of our ATAC-seq profile provided further evidence of direct occupancy of a TF candidate on genomic DNA and thus refined the prediction of potential regulators. We illustrated the "footprints" of 2

232 known regulators, STAT5 and T-bet, and observed higher DNA accessibility and deeper "footprints" flanking their motifs in the late compared with the early stage during NK cell differentiation (Figure 3D). Similarly, "footprints" of the TFs FOSL2 and EGR2 were also deeper and more accessible at the late stage, suggesting not only that the motifs of these 2 TFs were enriched at stage-specific peaks but that they were most likely physically bound to those accessible chromatin sites, indicating that they are functional regulators of NK differentiation (Figure 3D).

238 Overall, the results from footprint analysis were also consistent with that from the HOMER and Genomica’s motif enrichment analysis.

By combining the TF motif and "footprint" analysis, we can theoretically predict genes 241 that are regulated by any TF of interest. We performed this analysis on the TFs RUNX, T-bet, 242 FOSL2 and EGR2 and integrated the gene expression profiles at each time point (Figure S5B, 243 Table S2). We found that genes regulated by each of these TFs were also highly expressed at late 244 stages and were significantly enriched in GO terms of immune system construction and other related functions. Transcription factors ETS1 was reported to drive early stages of NK cell 
differentiation [53], and we found that there was a FOSL2 binding site in a dynamical accessible sites on the gene body of ETS1, suggesting FOSL2 might regulate NK cell differentiation through ETS1 (Figure 3E). Transcription factors GATA3 was found regulating liver-specific NK cells, IFN- $\gamma$ production, and T-bet expression in mice [54]. Similarly, we found that both FOSL2 and EGR2 bind to the gene body of GATA3 (Figure 3E), suggesting that the TFs FOSL2 and EGR2 may also regulate NK cell differentiation through regulation of GATA3.

FOSL2 and EGR2 are necessary for NK cell differentiation

Fosl2 belongs to the Fos gene family, which encodes leucine zipper proteins that combines to the TF complex AP-1 in the form of a dimer with JUN family. Thus, FOS proteins have been suggested as key regulators of transformation, differentiation and cell proliferation. FOSL2 (FOSlike 2) is a protein-coding gene. The GO annotations of FOSL2 include sequence-specific DNA binding, transcription factor activity and RNA polymerase II specific DNA binding. A previous report has shown that FOSL2 is constitutively expressed in adult T-cell leukemia (ATL) and upregulates CCR4 and promotes ATL cell growth, together with JUND [55]. EGR2 (early growth response 2) is also a protein-coding gene that contains three tandem $\mathrm{C} 2 \mathrm{H} 2$-type zinc fingers. $\mathrm{GO}$ annotations of this gene include ligase activity, sequence-specific DNA binding and transcription factor activity. Previous reports have shown that Egr2 regulate T-cell and B-cell function in homeostasis and adaptive immune responses by controlling inflammation and promoting antigen receptor signaling $[56,57]$.

Since their regulatory functions in NK cell differentiation have not been well characterized, we performed loss-of-function experiments to assess the effects of FOSL2 and EGR2 on NK cell differentiation. We first validated their expression levels with real-time PCR, and we found that

272 specific shRNA- and control shRNA-containing lentivirus, respectively, which are represented by

273 GFP expression (Figure 4A). We then sorted the GFP-positive cells and observed dramatically 274 reduced FOSL2 and EGR2 expression levels (Figure 4B), suggesting positive targeting of the TF275 specific shRNA. During NK cell differentiation, we observed a nearly $30 \%$ reduction in the proportion of differentiated NK cells in GFP-positive cells at days 28 and 35 (Figure 4C-D). 
277 However, in the non-transfected (GFP-negative) cells, the proportion of differentiated NK cells

278 was not affected at the same time (Figure 4E-F). These results indicate that knockdown of FOSL2

279 and EGR2 expression, but not viral infection, inhibit NK cell differentiation, suggesting that

280 FOSL2 and EGR2 are necessary for NK cell differentiation. We then tested another important

281 marker of NK cell maturation CD11b [1], and found that it was significantly reduced in GFP-

282 positive NK cells, suggesting that FOSL2 and EGR2 might affect NK cell maturation (Figure 4G).

283 Overall, we predicted these two TFs FOSL2 and EGR2 as key regulators based on ATAC-seq and

284 gene microarray profile analysis, and then experimentally verified that they were indeed necessary

285 for normal NK cell differentiation.

286 In addition, we attempted to identify the signaling pathways through which FOSL2 and

287 EGR2 were involved in driving NK cell differentiation by performing module map analysis of all

288 the differential peaks. 18 modules were identified according to the patterns of their accessibility

289 (Table S3), several of which were significantly enriched with known TFs that regulate NK cell

290 differentiation (Figure S6B), including FOSL2 and EGR2, and many genes in the JAK-STAT

291 pathway, such as the STAT family, IRF2, TBX21, and GATA3. These results suggest that FOSL2

292 and EGR2, may regulate NK cell differentiation through the JAK-STAT pathway (Figure S6C).

\section{Transcriptional regulatory network dynamics during NK cell differentiation}

The dramatic chromatin accessibility differences during NK cell differentiation prompted us to check the time point-specific transcriptional regulatory network. Although some TFs have been discovered to regulate NK cell differentiation, little is known about the dynamics of the entire regulatory network during this process. Since the TF footprint pattern from the ATAC-seq reads can simultaneously directly reveal the binding profiles of hundreds of TFs with known cognate motifs [22], together with gene expression profiles, we can construct a regulatory network at each time point and assess how it changes during NK cell differentiation. First, we used HOMER to identify enriched TFs that bound to the cluster I and cluster II peaks shown in Figure $3 \mathbf{A}(\mathrm{P}<0.05)$. We then examined the gene expression profiles of these TFs and found that 120 TFs were expressed in at least one stage during the differentiated process. By applying differential analysis, we obtained 14-26 TFs that were distinctly expressed at each stage (fold change>1.5) (Figure S7), and defined them as the nodes of the regulatory network. The connections (edges) between any 2 
TFs were defined as follows: If the TF A motif is on the promoter of TF B, then we say TF A regulates TF B and draw an arrow from TF A to TF B. Here, only TFs that were expressed at the specific time point were consideration [58]. Using this method, we constructed the transcriptional regulatory network at each time points with both the enrichment (P-value) and expression

312 information for all the relevant TFs (Figure 5A-E). Interestingly, day 7 specific TFs were densely interconnected at the beginning, and quickly vanished after two weeks (Figure 5A). In contrast, the day 35 specific network gradually grew out through the induction of relevant TFs. Many known regulators, such as EOMES, TBX21, ETS1, PRDM1, and GATA3 and also FOLS2 and EGR2 were increasingly enriched on the network (Figure 5E). Similar phenomena were also observed on other networks (Figure 5B-D). We believe the dynamics of the transcriptional regulatory network explain the increase in the proportion and the differentiation of NK cells.

\section{Discussion}

NK cells are important innate immune cells that have been recognized as efficient effector cells to treat tumors. To better understand the differentiation machinery of NK cells and identify new regulators in the process, we studied the landscape of active elements during NK cell differentiation using the sensitive ATAC-seq method. As shown by the ATAC-seq results, three distinct clusters of DNA accessible elements were found. Further analysis showed that the chromatin accessibility correlated well with the level of the expression of the corresponding genes. In short, this study provides an epigenomic landscape and dynamics of NK cell differentiation and presents foundational profiles for studying the relationship between chromatin accessibility, gene expression and cell growth during this process (Figure 2).

TFs bind to their motifs and are often obligate nucleosome evictors and the creators of accessible DNA sites, and therefore we can use ATAC-seq to predict critical regulators in NK cell differentiation [26]. By motif analysis in HOMER, we found that several known TFs were enriched

334 at different stages during NK cell differentiation. Similar results were also obtained using 335 Genomica (Figure 3B). The discovery of known regulators strongly suggested the reliability of 336 our analysis Furthermore, by integrating results from HOMER, Genomica and motif footprint 337 analysis, we identified two novel TFs, FOSL2 and EGR2 that were essential for NK cell maturation. 
NK cell differentiation system. Module map analysis suggested these two TFs may regulate NK cell through the JAK-STAT pathway, and therefore further studies of this pathway may facilitate the generation of NK cells and thus promote the NK cell-based immunotherapy. Overall, this study also provided a framework to identify new regulators from chromatin accessible data for NK cell differentiation.

TFs do not usually function alone, they always interact with other molecules to fulfill their unique roles. Hence, we depicted the transcriptional regulatory networks at different stages during NK cell differentiation. In order to construct a stable transcriptional regulatory network, we performed a rigorous screening of TFs to avoid stochastic fluctuations, and integrated both the enrichment (P-value) and expression information for all the relevant TFs. Therefore, even a change of either the enrichment or gene expression cutoff may result in different networks, the most critical TFs to the regulatory process still remain. However, since the screening of TFs mainly rely on the gene microarray, which is not as accurate as RNA-seq, the structure of these predicted network may not as robust as well.

From our previous study, we noticed that with a minimal cytokine cocktail, we can generate sufficient number of functional NK cells that express the cytokines necessary for NK cells and have a high effect on tumor cells [19]. Although there may also be a certain proportion of other lymphocytes, in vitro expansion of NK cells from peripheral blood (PB) or UCB cells has been successfully performed and developed in several clinical strategies to treat cancer [4, 5, 59-61]. Therefore, a comprehensive understanding of the regulatory patterns at each differentiated stage of the in vitro-derived NK cells in this system will help to uncover the underlying mechanisms of NK cell differentiation. The transcriptional regulatory network revealed in this study will lay the foundation for faster and better in vitro production of effective NK cells, thus facilitating NK cellbased immunotherapy. Moreover, we have identified two novel TFs, FOSL2 and EGR2, as essential regulators in controlling NK cell maturation and function. We also predicted the potential signaling pathways in which these two TFs may be involved and illustrated the dynamics of the transcriptional regulatory network during NK cell differentiation. In spite of the advantages of our strategy, there are two main limitations of this study: (1) Although induced NK cells are very similar to those produced in vivo, these two types of NK cells are still not identical. We observed certain differences between the induced NK cells and the NK cells produced in vivo in terms of 
370 a mixture of cell populations with stem cells, NK progenitor cells, immature NK cells and mature

371 NK cells and other cell types that we are incapable to delineate at this moment, i.e. any bulk cell

372 based analysis may neglect the huge heterogeneity between cells by default. Therefore, to fully

373 uncover the regulatory mechanism, single cell technologies are required in the future to further

374 delineate the cell-to-cell heterogeneity and regulatory dynamics at single cell precision.

Materials and Methods

Samples: Umbilical cord blood (UCB) samples were collected at birth from women with normal, full-term deliveries at Anhui Provincial Hospital, Hefei, after receiving informed consent. Approval was obtained from the Ethics Committee of the University of Science and Technology of China. The culture procedure for NK cell differentiation from UCB CD $34^{+}$cells has been previously described [19].

Immunofluorescence staining: The cultured cells were post-fixed in $4 \%$ paraformaldehyde, blocked with $10 \%$ goat serum and stained with primary antibodies at $4{ }^{\circ} \mathrm{C}$ overnight. Secondary antibodies were stained at $37^{\circ} \mathrm{C}$ for $1 \mathrm{~h}$. Cell nuclei were stained with DAPI for 5 min at room temperature. Confocal images were acquired using a Zeiss LS710 microscope.

RNA isolation and real-time PCR: Cultured cells were lysed in TRIzol reagent (Invitrogen), and total RNA was extracted following the manufacturer's instructions. cDNA was synthesized with Moloney murine leukemia virus reverse transcriptase (Invitrogen) and oligo (dT) 20 primers. Then, SYBR Premix Ex Taq (TaKaRa) was used for real-time PCR (primer sequences, Table S4). The data were analyzed using the $2-\Delta \Delta \mathrm{Ct}$ method.

392 Lentivirus production and transduction: To produce lentiviral particles, 293T cells were 393 transfected with the plasmids PLKO.1, pRRE, pREV and pVSV-G via Lipofectamine 2000 394 (Invitrogen) following the manufacturer's protocol. Then, we harvested the supernatants 48 and $39572 \mathrm{~h}$ post-transfection. To remove cell debris, supernatants were centrifuged (3000 rpm, $10 \mathrm{~min}$ ), 396 and then, the lentivirus particles were concentrated by ultracentrifugation at $50000 \mathrm{~g}$ for $2 \mathrm{~h}$ at $4^{\circ} \mathrm{C}$. 397 Finally, the virus particles were gently resuspended in $\mathrm{HBSS}$ and stored at $-80^{\circ} \mathrm{C}$. After UCB $398 \mathrm{CD}^{+} 4^{+}$cells were cultured with multiple cytokines for 14-18 d, we incubated the lentivirus and the 399 cultured cells with polybrene $(5 \mu \mathrm{g} / \mathrm{ml})$ and centrifuged them at $1000 \mathrm{rpm}$ for $70 \mathrm{~min}$ at $10^{\circ} \mathrm{C}$. 
Statistical analysis: Statistical significance was analyzed using unpaired two-tailed t-tests. Pvalues less than 0.05 were considered statistically significant.

402 Microarray: The microarray was performed using an Affymetrix GeneChip ${ }^{\circledR}$ PrimeView Human

403 Gene Expression Array. The signal values of the samples were normalized using RMA.

404 Differential analysis was performed using Student's t-test as previously described [19]. The microarray data were under accession number GEO: GSE87787. ATAC-seq: ATAC-seq was performed as previously described [22], and 2x150 paired-end sequencing was performed on an Illumina HiSeq X-10 to yield, on average, $78 \mathrm{M}$ reads/sample. ATAC-seq analysis: Sample reads from biological replicates were then grouped together and divided into 8 categories: day 7, day 14, day19, day 21, day 24, day 26, day 28 and day 35. Intrinsic analysis and other ATAC-seq analysis was performed same as our previous work $[26,62]$. Difference analysis: All samples were grouped into 8 categories (16 samples): day 7, 14, 19, 21, 24, 26, 28 and day 35. Data normalization and significance analysis were performed via pairwise comparison between the 8 categories using DESeq2 [63], with a $\mathrm{P}<0.01$, log2-fold change $>5$, $\mathrm{FDR}<0.01$, and intrinsic analysis [26] and with a z-score $>1$. We finally obtained 6401 differential peaks. Unsupervised clustering was performed using Cluster 3.0 and visualized in Treeview. GREAT [33] was used to predict enriched functions and Gene Ontology.

417 Stage-specific peaks: Each stage (e.g., day 7) consists of genes that were induced (>1.5-fold 418 change) at that stage compared with all the other stages. We defined peaks that were accessible 419 only in one stage as stage-specific peaks, and those that were accessible at all stages as 420 conservative peaks.

421 Define promoter: We define the range of $2 \mathrm{~kb}$ around the transcription start site as a promoter.

422 TF motif enrichment analysis: HOMER [29] was used to perform the TF enrichment analysis 423 with the following options: findmotifs.pl input.fa fasta output/ for Figure 3A and findmotifs.pl 424 input.fa human uotputdir -fasta bg.fa for Figure S5A. TF enrichment analysis using Genomica 425 and TF footprinting analysis was performed the same as our previous work [26].

426 Gene Module Analysis: Gene module analyses were performed using WGCNA [64] in Figure 427 S6B with the options SoftPower $=20$, minModuleSize $=30$.

428 STRING analysis: Protein-protein interaction analyses were performed using STRING [65] in 429 Figure S6C. 
Transcriptional regulatory network: We used HOMER to find the transcription factors that bind to cluster I and cluster II and obtained a total of 253 transcription factors that could regulate these 432 differentially expressed elements $(\mathrm{P}<0.05)$, of which 120 were expressed in the microarray profile 433 of the corresponding sample. If TF A bound to the promoter of TF B, we defined TF A as a 434 regulator of TF B and then constructed a transcriptional regulatory network. Networks of TFs were assembled from this set of $120 \mathrm{TFs}$ that were expressed in at least one sample. An edge between TF A and TF B indicated that TF A bound to the promoter of TF B [58].

Data Availability

GSA accession: The raw sequence data have been deposited in the Genome Sequence Archive [66] in BIG Data Center [67], under the accession number CRA000846 and are publicly accessible 442 at http://bigd.big.ac.cn/gsa/s/u7fdeNV3.

\section{Acknowledgements}

This work was supported by the National Natural Science Foundation of China grant 81788101 447 (to Z.T.), the National Key R\&D Program of China 2017YFA0102903 (to K.Q.), the National 448 Natural Science Foundation of China grant 91640113 (to K.Q.) and 31771428 (to K.Q.), the key 449 project of Natural Science Foundation of China 81330071 (to H.W.). We thank the USTC 450 supercomputing center and the School of Life Science Bioinformatics Center for providing 451 supercomputing resources for this project.

\section{Authors' contributions}

KQ and HW conceived the project, YW and YL performed all the cell sorting and ATAC-seq 456 library experiments, KL performed most of the data analysis, QY analyzed the microarray data, 457 HW and ZT discussed the results and provided advices, KL and KQ wrote the manuscript.

\section{Conflict interests}


All authors have no competing financial interests.

\section{References}

[1] Sun JC. Transcriptional Control of NK Cells. Curr Top Microbiol Immunol 2016;395:1-36. [2] Miller JS, Soignier Y, Panoskaltsis-Mortari A, McNearney SA, Yun GH, Fautsch SK, et al. Successful adoptive transfer and in vivo expansion of human haploidentical NK cells in patients with cancer. Blood 2005;105:3051-7.

[3] Miller JS, Verfaillie C, McGlave P. The generation of human natural killer cells from CD34+/DR- primitive progenitors in long-term bone marrow culture. Blood 1992;80:2182-7.

[4] Luevano M, Madrigal A, Saudemont A. Generation of natural killer cells from hematopoietic stem cells in vitro for immunotherapy. Cell Mol Immunol 2012;9:310-20.

[5] Cheng M, Chen Y, Xiao W, Sun R, Tian Z. NK cell-based immunotherapy for malignant diseases. Cell Mol Immunol 2013;10:230-52.

[6] Dolstra H, Roeven MWH, Spanholtz J, Hangalapura BN, Tordoir M, Maas F, et al. Successful Transfer of Umbilical Cord Blood CD34(+) Hematopoietic Stem and Progenitor-derived NK Cells in Older Acute Myeloid Leukemia Patients. Clin Cancer Res 2017;23:4107-18.

[7] Ruggeri L, Capanni M, Urbani E, Perruccio K, Shlomchik WD, Tosti A, et al. Effectiveness of donor natural killer cell alloreactivity in mismatched hematopoietic transplants. Science 2002;295:2097-100.

[8] Guillerey C, Huntington ND, Smyth MJ. Targeting natural killer cells in cancer immunotherapy. Nat Immunol 2016;17:1025-36.

[9] Sivori S, Cantoni C, Parolini S, Marcenaro E, Conte R, Moretta L, et al. IL-21 induces both rapid maturation of human CD34+ cell precursors towards NK cells and acquisition of surface killer Ig-like receptors. Eur J Immunol 2003;33:3439-47.

[10] Spanholtz J, Tordoir M, Eissens D, Preijers F, van der Meer A, Joosten I, et al. High log-scale expansion of functional human natural killer cells from umbilical cord blood CD34-positive cells for adoptive cancer immunotherapy. PLoS One 2010;5:e9221.

[11] Kao IT, Yao CL, Kong ZL, Wu ML, Chuang TL, Hwang SM. Generation of natural killer cells from serum-free, expanded human umbilical cord blood CD34+ cells. Stem Cells Dev 2007;16:1043-51.

[12] Haraguchi K, Suzuki T, Koyama N, Kumano K, Nakahara F, Matsumoto A, et al. Notch activation induces the generation of functional NK cells from human cord blood CD34-positive cells devoid of IL-15. J Immunol 2009;182:6168-78.

[13] Yoon SR, Lee YS, Yang SH, Ahn KH, Lee JH, Lee JH, et al. Generation of donor natural killer cells from CD34(+) progenitor cells and subsequent infusion after HLA-mismatched allogeneic hematopoietic cell transplantation: a feasibility study. Bone Marrow Transplant 2010;45:1038-46.

[14] Shah N, Martin-Antonio B, Yang H, Ku S, Lee DA, Cooper LJ, et al. Antigen presenting cellmediated expansion of human umbilical cord blood yields log-scale expansion of natural killer cells with anti-myeloma activity. PLoS One 2013;8:e76781.

[15] Vitale C, Cottalasso F, Montaldo E, Moretta L, Mingari MC. Methylprednisolone induces preferential and rapid differentiation of CD34+ cord blood precursors toward NK cells. Int Immunol 2008;20:565-75. 
[16] Carayol G, Robin C, Bourhis JH, Bennaceur-Griscelli A, Chouaib S, Coulombel L, et al. NK cells differentiated from bone marrow, cord blood and peripheral blood stem cells exhibit similar phenotype and functions. Eur J Immunol 1998;28:1991-2002. [17] Yu Y, Hagihara M, Ando K, Gansuvd B, Matsuzawa H, Tsuchiya T, et al. Enhancement of human cord blood CD34+ cell-derived NK cell cytotoxicity by dendritic cells. J Immunol 2001;166:1590-600. [18] Cany J, van der Waart AB, Spanholtz J, Tordoir M, Jansen JH, van der Voort R, et al. Combined IL-15 and IL-12 drives the generation of CD34(+)-derived natural killer cells with superior maturation and alloreactivity potential following adoptive transfer. Oncoimmunology 2015;4:e1017701. cells kill leukemia cells by engaging SLAM family receptors. Oncotarget 2017;8:57024-38. [20] Colucci F, Samson SI, DeKoter RP, Lantz O, Singh H, Di Santo JP. Differential requirement for the transcription factor PU.1 in the generation of natural killer cells versus B and T cells. Blood 2001;97:2625-32.

[21] Gordon SM, Chaix J, Rupp LJ, Wu J, Madera S, Sun JC, et al. The transcription factors T-bet and Eomes control key checkpoints of natural killer cell maturation. Immunity 2012;36:55-67.

[22] Buenrostro JD, Giresi PG, Zaba LC, Chang HY, Greenleaf WJ. Transposition of native chromatin for fast and sensitive epigenomic profiling of open chromatin, DNA-binding proteins and nucleosome position. Nat Methods 2013;10:1213-8.

[23] Sen DR, Kaminski J, Barnitz RA, Kurachi M, Gerdemann U, Yates KB, et al. The epigenetic landscape of T cell exhaustion. Science 2016;354:1165-9.

[24] Pauken KE, Sammons MA, Odorizzi PM, Manne S, Godec J, Khan O, et al. Epigenetic stability of exhausted $\mathrm{T}$ cells limits durability of reinvigoration by PD-1 blockade. Science 2016;354:1160-5. [25] Denny SK, Yang D, Chuang CH, Brady JJ, Lim JS, Gruner BM, et al. Nfib Promotes Metastasis through a Widespread Increase in Chromatin Accessibility. Cell 2016;166:328-42.

[26] Qu K, Zaba LC, Giresi PG, Li R, Longmire M, Kim YH, et al. Individuality and variation of personal regulomes in primary human T cells. Cell Syst 2015;1:51-61.

[27] Qu K, Zaba LC, Satpathy AT, Giresi PG, Li R, Jin Y, et al. Chromatin Accessibility Landscape of Cutaneous T Cell Lymphoma and Dynamic Response to HDAC Inhibitors. Cancer Cell 2017;32:27-41 e4.

[28] Lau CM, Adams NM, Geary CD, Weizman OE, Rapp M, Pritykin Y, et al. Epigenetic control of innate and adaptive immune memory. Nat Immunol 2018;19:963-72.

[29] Heinz E, Born D, Zieger G, May T, Krause T, Kruger A, et al. Progress report on Safe VISITOR: approaching a practical instrument for terahertz security screening. Passive MillimeterWave Imaging Technology Xiii 2010;7670.

[30] Yu J, Freud AG, Caligiuri MA. Location and cellular stages of natural killer cell development. Trends Immunol 2013;34:573-82.

[31] Montaldo E, Del Zotto G, Della Chiesa M, Mingari MC, Moretta A, De Maria A, et al. Human NK cell receptors/markers: a tool to analyze NK cell development, subsets and function. Cytometry A 2013;83:702-13.

[32] Perou CM, Sorlie T, Eisen MB, van de Rijn M, Jeffrey SS, Rees CA, et al. Molecular portraits of human breast tumours. Nature 2000;406:747-52.

550

[33] McLean CY, Bristor D, Hiller M, Clarke SL, Schaar BT, Lowe CB, et al. GREAT improves functional interpretation of cis-regulatory regions. Nature Biotechnology 2010;28:495-U155. 
[34] Grewal SI, Moazed D. Heterochromatin and epigenetic control of gene expression. Science 2003;301:798-802.

[35] Ohno S, Sato T, Kohu K, Takeda K, Okumura K, Satake M, et al. Runx proteins are involved in regulation of CD122, Ly49 family and IFN-gamma expression during NK cell differentiation. Int Immunol 2008;20:71-9.

[36] Yamanaka R, Lekstrom-Himes J, Barlow C, Wynshaw-Boris A, Xanthopoulos KG. CCAAT/enhancer binding proteins are critical components of the transcriptional regulation of hematopoiesis (Review). Int J Mol Med 1998;1:213-21.

[37] Chang HC, Zhang S, Thieu VT, Slee RB, Bruns HA, Laribee RN, et al. PU.1 expression delineates heterogeneity in primary Th2 cells. Immunity 2005;22:693-703.

[38] Dakic A, Metcalf D, Di Rago L, Mifsud S, Wu L, Nutt SL. PU.1 regulates the commitment of adult hematopoietic progenitors and restricts granulopoiesis. J Exp Med 2005;201:1487-502.

[39] Nutt SL, Metcalf D, D'Amico A, Polli M, Wu L. Dynamic regulation of PU.1 expression in multipotent hematopoietic progenitors. J Exp Med 2005;201:221-31.

[40] Carotta S, Dakic A, D'Amico A, Pang SH, Greig KT, Nutt SL, et al. The transcription factor PU.1 controls dendritic cell development and Flt3 cytokine receptor expression in a dosedependent manner. Immunity 2010;32:628-41.

[41] Iwasaki H, Somoza C, Shigematsu H, Duprez EA, Iwasaki-Arai J, Mizuno S, et al. Distinctive and indispensable roles of PU.1 in maintenance of hematopoietic stem cells and their differentiation. Blood 2005;106:1590-600.

[42] Boos MD, Yokota Y, Eberl G, Kee BL. Mature natural killer cell and lymphoid tissueinducing cell development requires Id2-mediated suppression of E protein activity. Journal of Experimental Medicine 2007;204:1119-30.

[43] Eckelhart E, Warsch W, Zebedin E, Simma O, Stoiber D, Kolbe T, et al. A novel Ncr1-Cre mouse reveals the essential role of STAT5 for NK-cell survival and development. Blood 2011;117:1565-73.

[44] Collins A, Littman DR, Taniuchi I. RUNX proteins in transcription factor networks that regulate T-cell lineage choice. Nat Rev Immunol 2009;9:106-15.

[45] Mathelier A, Fornes O, Arenillas DJ, Chen CY, Denay G, Lee J, et al. JASPAR 2016: a major expansion and update of the open-access database of transcription factor binding profiles. Nucleic Acids Res 2016;44:D110-5.

[46] O'Shea JJ, Schwartz DM, Villarino AV, Gadina M, McInnes IB, Laurence A. The JAK-STAT pathway: impact on human disease and therapeutic intervention. Annu Rev Med 2015;66:311-28. [47] Tamura T, Yanai H, Savitsky D, Taniguchi T. The IRF family transcription factors in immunity and oncogenesis. Annu Rev Immunol 2008;26:535-84.

[48] Taniguchi T, Ogasawara K, Takaoka A, Tanaka N. IRF family of transcription factors as regulators of host defense. Annual Review of Immunology 2001;19:623-55.

[49] Lohoff M, Duncan GS, Ferrick D, Mittrucker HW, Bischof S, Prechtl S, et al. Deficiency in the transcription factor interferon regulatory factor (IRF)-2 leads to severely compromised development of natural killer and T helper type 1 cells. J Exp Med 2000;192:325-36.

[50] Taki S, Nakajima S, Ichikawa E, Saito T, Hida S. IFN Regulatory Factor-2 Deficiency Revealed a Novel Checkpoint Critical for the Generation of Peripheral NK Cells. The Journal of Immunology 2005;174:6005-12.

[51] Szabo SJ, Kim ST, Costa GL, Zhang X, Fathman CG, Glimcher LH. A novel transcription factor, T-bet, directs Th1 lineage commitment. Cell 2000;100:655-69. 
[52] Townsend MJ, Weinmann AS, Matsuda JL, Salomon R, Farnham PJ, Biron CA, et al. T-bet regulates the terminal maturation and homeostasis of $\mathrm{NK}$ and $\mathrm{V}$ alpha $14 \mathrm{i} \mathrm{NKT}$ cells. Immunity 2004;20:477-94.

[53] Ramirez K, Chandler KJ, Spaulding C, Zandi S, Sigvardsson M, Graves BJ, et al. Gene deregulation and chronic activation in natural killer cells deficient in the transcription factor ETS1. Immunity 2012;36:921-32.

[54] Samson SI, Richard O, Tavian M, Ranson T, Vosshenrich CA, Colucci F, et al. GATA-3 promotes maturation, IFN-gamma production, and liver-specific homing of NK cells. Immunity 2003;19:701-11.

[55] Higuchi T, Nakayama T, Arao T, Nishio K, Yoshie O. SOX4 is a direct target gene of FRA2 and induces expression of HDAC8 in adult T-cell leukemia/lymphoma. Blood 2013;121:36409.

[56] Li S, Miao T, Sebastian M, Bhullar P, Ghaffari E, Liu M, et al. The transcription factors Egr2 and Egr3 are essential for the control of inflammation and antigen-induced proliferation of $\mathrm{B}$ and T cells. Immunity 2012;37:685-96.

[57] Okamura T, Fujio K, Sumitomo S, Yamamoto K. Roles of LAG3 and EGR2 in regulatory T cells. Ann Rheum Dis 2012;71 Suppl 2:i96-100.

[58] Novershtern N, Subramanian A, Lawton LN, Mak RH, Haining WN, McConkey ME, et al. Densely interconnected transcriptional circuits control cell states in human hematopoiesis. Cell 2011;144:296-309.

[59] Suck G, Koh MB. Emerging natural killer cell immunotherapies: large-scale ex vivo production of highly potent anticancer effectors. Hematol Oncol Stem Cell Ther 2010;3:135-42.

[60] Smyth MJ, Hayakawa Y, Takeda K, Yagita H. New aspects of natural-killer-cell surveillance and therapy of cancer. Nat Rev Cancer 2002;2:850-61.

[61] Fujisaki H, Kakuda H, Shimasaki N, Imai C, Ma J, Lockey T, et al. Expansion of highly cytotoxic human natural killer cells for cancer cell therapy. Cancer Res 2009;69:4010-7.

[62] Zuo Z, Jin Y, Zhang W, Lu Y, Li B, Qu K. ATAC-pipe: general analysis of genome-wide chromatin accessibility. Brief Bioinform 2018.

[63] Anders S, Huber W. Differential expression analysis for sequence count data. Genome Biology 2010;11.

[64] Langfelder P, Horvath S. WGCNA: an R package for weighted correlation network analysis. BMC Bioinformatics 2008;9:559.

[65] Snel B, Lehmann G, Bork P, Huynen MA. STRING: a web-server to retrieve and display the repeatedly occurring neighbourhood of a gene. Nucleic Acids Res 2000;28:3442-4.

[66] Wang Y, Song F, Zhu J, Zhang S, Yang Y, Chen T, et al. GSA: Genome Sequence Archive. Genomics Proteomics Bioinformatics 2017;15:14-8.

[67] Members BIGDC. Database Resources of the BIG Data Center in 2018. Nucleic Acids Res 2018;46:D14-D20. 


\section{Figures legends}

636

637

Figure 1. Landscape of DNA accessibility during NK cell differentiation

638 A Confocal microscopy images of membrane CD56 (red) in the cultured cells at days 7, 14, 21, 63928 and 35. Scale bars, $30 \mu \mathrm{m}$. Nuclei are stained with DAPI.

640 B Schematic representation of the overall experimental design of this study. Gene expression and 641 chromosome opening at different time points were assessed using microarray and ATAC-seq data 642 respectively. The bioinformatics pipeline for data analysis is shown in the bottom.

$643 \mathrm{C}$ The gene expression profiles of SPI1 and TBX21 at different stages of NK cell differentiation.

644 D Flow cytometric measurement of PU.1 (SPI1-encoded protein) and T-bet (TBX21-encoded 645 protein) expression using in cultured cells during a 35-day time course.

646 E Normalized ATAC-seq profiles of the SPI1 (top) and TBX21 (bottom) gene loci at different 647 stages during NK cell differentiation. These two genes are known to regulate NK cell 648 differentiation, and ATAC-seq signals were obtained from the UCSC genome browser.

651 A Heatmap of 6401 differentially expressed regulatory elements during NK cell differentiation. 652 Each column is a sample, and each row is a peak. Samples and peaks are organized by two653 dimensional unsupervised hierarchical clustering. Color scale showing the relative ATAC-seq 654 peak intensity centered by each peak summit. Top: samples at all time points were categorized into 655 three groups, early stage: days 7 21 (orange); interim stage: days 24 28 (yellow) and late stage: 656 days 28 35 (green). Samples from the same cluster are labeled with the same color. Left: 657 differential peaks are categorized into 3 clusters.

658 B The top ten most significant gene ontology (GO) terms enriched in cluster I and cluster II peaks.

659 C Box-plots of the mRNA expression levels of the genes that are more accessible in early, interim 660 and late stages during NK cell differentiation. 1,2 represents biological replicates 1 and 2, 661 respectively. P-values were estimated from Student's t-test.

662 D The changes in ATAC-seq signals (red), gene expression (orange) and number of NK cells 663 (green) at different time points during NK cell differentiation. 
666

667

668

669

670

671

672

673

674

675

676

677

678

679

680

681

682

683

684

685

686

687

688

689

690

691

692

693

694 $695 \quad(\mathrm{n}=5)$.

696

A TF motifs enriched in cluster I (left) and cluster II (right) peaks, with enrichment P-values estimated from HOMER. TFs known to regulate NK cell differentiation are colored red.

B Enrichment of known TF motifs in differential accessible elements in all samples. Each row is a motif, and each column is a sample. Values in the matrix represent the significance level in terms of the $-\log (\mathrm{P}$-value $)$ of the enrichment estimated from Genomica. Red indicates that the motif is enriched in the corresponding sample, and blue indicates depletion. Red box indicates known key TFs that regulates NK cell development, the pink box indicates the new TF whose function will be experimentally tested later in Figure 4.

C Prediction of TFs that may regulate NK cell differentiation. TFs known to regulate NK cell differentiation are shown at the top, TFs predicted to regulate early stages of the differentiation process are shown in the bottom, and those that regulate the late stage are shown at the middle. The color of each circle represents the expression level of the gene encoding the corresponding $\mathrm{TF}$, while the size of the circle represents the significance of the motifs estimated by P-values.

D Visualization of the ATAC-seq footprint for STAT5, TBET, FOSL2 and EGR2 motifs during five stages of NK cell differentiation: day 7 (blue), day 14 (green), day 21 (red), day 28 (cyan), day 35 (purple). ATAC-seq signals across all these motif binding sites in the genome were aligned on the motif and averaged.

E Normalized ATAC-seq profiles of the ETS1 (left) and GATA3 (right) gene loci at different stages during NK cell differentiation. These two genes are known to regulate NK cell differentiation, and ATAC-seq signals were obtained from the UCSC genome browser. Bottom: The motif of FOSL2 and EGR2.

\section{Figure 4. FOSL2 and EGR2 are necessary for NK cell differentiation}

A The gating strategy for cultured cells transduced with lentiviruses expressing either shFOSL2, shEGR2 or control shRNA via detection of GFP expression.

B Knockdown efficiency of FOSL2 and EGR2 with shFOSL2 and shEGR2.

C Flow cytometric analysis of CD56 in the shControl-, shFOSL2- and shEGR2-positive cells at days 28 and 35 .

D Quantification of CD56-positive cells in the GFP-positive cell population at days 28 and 35

E Flow cytometric analysis of CD56 in the GFP-negative cell population at days 28 and 35. 
F Quantification of CD56-positve cells in the GFP-negative cell population at days 28 and 35 $698(\mathrm{n}=5)$.

G Quantification of CD11b-positive cells in the $\mathrm{CD}^{+} 6^{+}$cell population at day $35(\mathrm{n}=5)$. The results

from five biological replicates are presented as the mean $\pm \mathrm{SEM} . * \mathrm{P}<0.05, * * \mathrm{P}<0.001$, and $* * * \mathrm{P}<0.0005$.

Figure 5. Transcriptional regulatory eetwork dynamics during NK cell differentiation

A $\sim$ E Cis-regulatory networks between TFs (nodes) enriched in at least one gene set and specifically expressed (fold change >1.5) at day 7 (A), day 14 (B), day 21 (C), day 28 (D) and day 35 (E). The arrow at the edge from node A to node B indicates that TF A regulates TF B by binding to the promoter site of the latter. The color of each node indicates the expression value of the gene encoding that $\mathrm{TF}$, and the size of the circle represents the significance of the motif enrichment according to the P-value. The types of edges indicate the Pearson correlation between the gene expression profiles of the connected TFs: positively correlated (correlation coefficient $>0.4$ ); negatively correlated (correlation coefficient $<-0.4$ ); no correlation (correlation coefficient between

$712-0.4$ to 0.4$)$.

Supplementary Figure S1. Landscape of DNA accessibility during NK cell differentiation

A Fractions of $\mathrm{CD} 6^{+} \mathrm{CD}^{-}$cells in the total gated cells during a 35-day time course.

717 B-C Quality control analysis of ATAC-seq data. B: The TSS enrichment score for all samples. C:

718 The fragment length distribution of all the mapped reads.

719 D Known cell surface markers: Normalized ATAC-seq profiles of the CD34, KIT(CD117), $720 K L R D 1(C D 94)$ and NCAM1(CD56) gene loci at different stages during NK cell differentiation.

721 E Normalized ATAC-seq profiles of the EOMES (left) and GATA3 (right) gene loci at different 722 stages during NK cell differentiation. These genes are known to regulate NK cell differentiation, 723 and ATAC-seq signals were obtained from the UCSC genome browser.

\section{Supplementary Figure S2. The correlation analysis on the samples}

726 A The Pearson correlation analysis on the replicates at each time point. R at the top is the Pearson correlation coefficient. 
B Heatmap of the Pearson correlation between all the samples with unsupervised clustering performed in Cluster 3.0.

\section{Supplementary Figure S3. Epigenomic signatures of NK cell differentiation at different}

732 stages

733 A Venn diagram of peaks identified at each stage of NK cell differentiation. Specific peaks were 734 defined as peaks that were identified only at a specific time point, and conserved peaks were those 735 identified at all stages during the process.

736 B The top most significant GO terms of all the stage-specific and conservative peaks.

Supplementary Figure S4. Epigenomic signatures of different stages during NK cell differentiation

741 sample, and each row is a peak. Samples and peaks were organized by two-dimensional 742 unsupervised hierarchical clustering. The color scale shows the relative ATAC-seq signal

743 intensities as indicated. Top: samples at all time points were categorized into three groups, early 744 stage: days 7 21 (orange); interim stage: days 24 28 (yellow) and late stage: days 28 35 (green).

745 Samples from the same cluster are labeled with the same color. Left: differential peaks are 746 categorized into 3 clusters.

747 B Distance from all the peaks in cluster I, II, and III to their nearest genes. Known TFs regulating $748 \quad$ NK cell differentiation are labeled.

749 C Principle component analysis of chromatin accessibility during NK cell differentiation. Three 750 clusters were also identified: early (days 7 21), interim (days $24 \sim 28$ ), and late (day 35).

752 Supplementary Figure S5. Transcription factor occupancy network during NK cell 753 differentiation

755 A The top ten TF motifs enriched in cluster I (left) and cluster II (right) peaks, with enrichment P756 values estimated from HOMER. TFs known to regulate NK cell differentiation are color-coded.

757 B Left: heatmaps of the gene expression profiles of the genes predicted to be regulated by RUNX, 758 T-bet, EGR2 and FOSL2. Unsupervised hierarchical clustering was performed. Right: the top ten 
759 most significant GO terms enriched in up-regulated (orange) and down-regulated (green) genes

760 predicted to be regulated by each TF on the left.

761

762 Supplementary Figure S6. EGR2 and FOSL2 are necessary for NK cell differentiation

763 A Real-time qPCR analysis (n=3) of the genes EGR2 (left) and FOSL2 (right). The results from

764 three replicates are presented as the mean \pm SEM.

765 B Module map analysis in Genomica: representative modules from module map analysis in

766 Genomica. Top: the most significantly enriched TFs in each module; bottom: the chromatin

767 accessibility changes of the peaks in the corresponding module compared with day 7. Right: genes

768 associated with peaks in that module.

769 C Signaling pathways of known and predicted TFs that regulate NK cell differentiation.

770

771 Supplementary Figure S7. Define time-specific TFs based on differential expression analysis

772 A: Distribution of all TF's fold change. $\mathrm{X}$ axis represents the fold change. $\mathrm{y}$ axis represents the

773 density of TF under different fold change. Std indicates Standard Deviation. Foldchange1.5 is 2

774 standard deviations away. Colors represent different time points.

775 B: The number of TFs that qualify fold changes. X axis represents different FDC (fold change).

776 The y axis represents the number of TFs that pass the fold change cutoff.

777 C: The ratio of known TFs versus all known TFs that qualify the different fold change cutoffs.

778 Known TFs: TFs regulating NK cell development reported in the literature. 
A

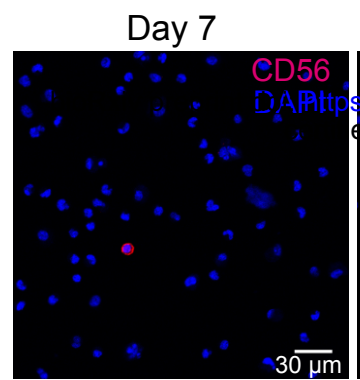

Day 14

Day 21
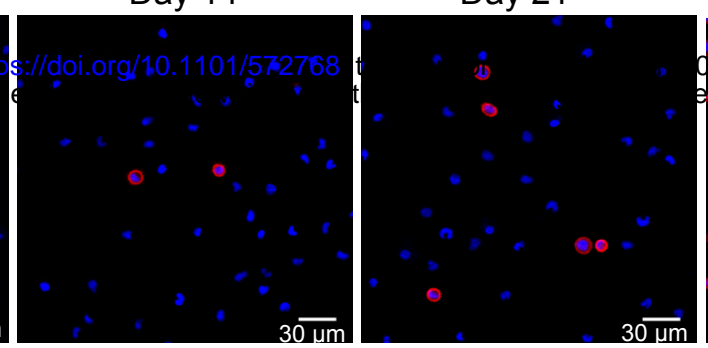

Day 28

Day 35
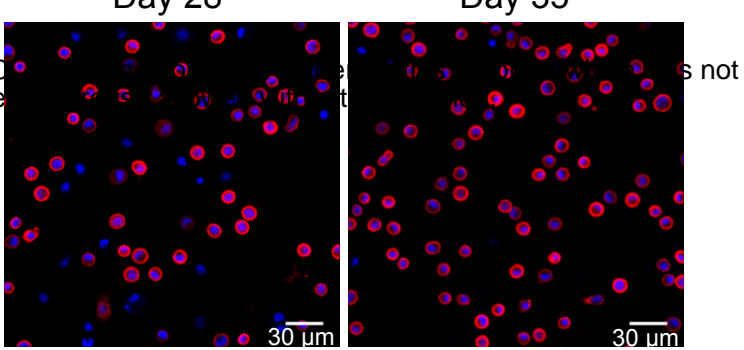

B
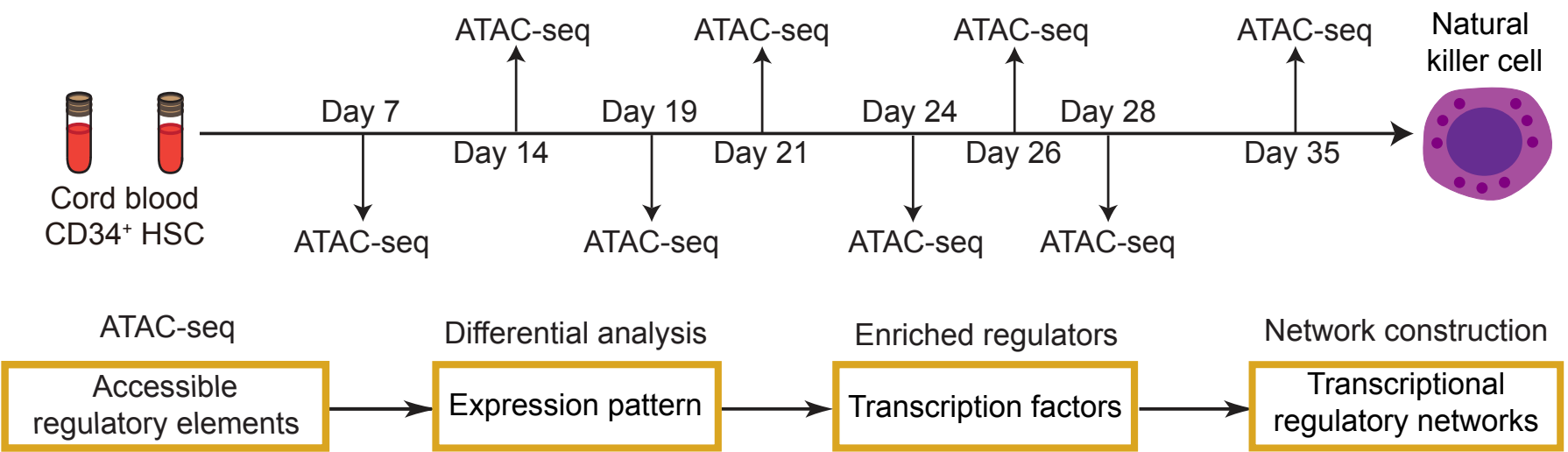

C

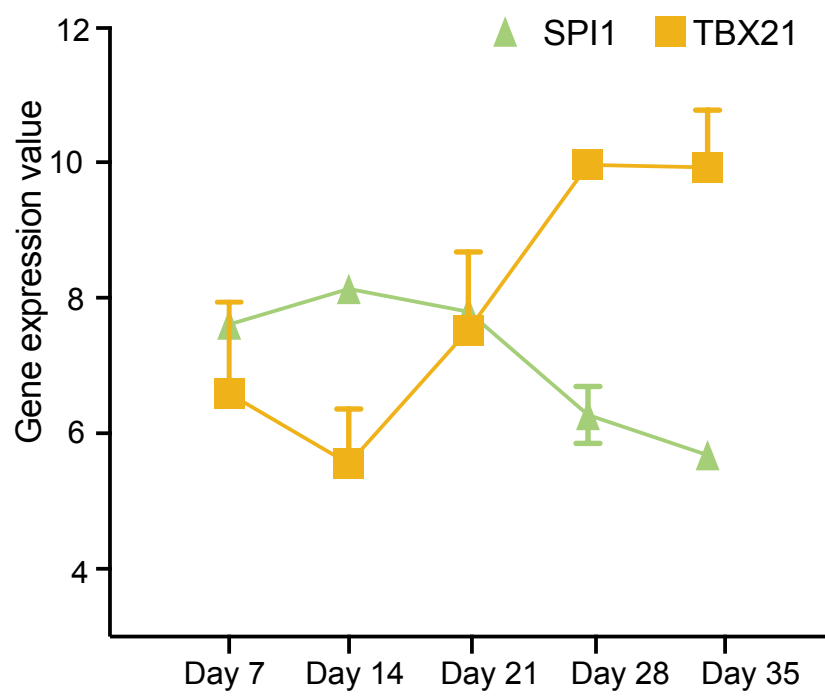

D

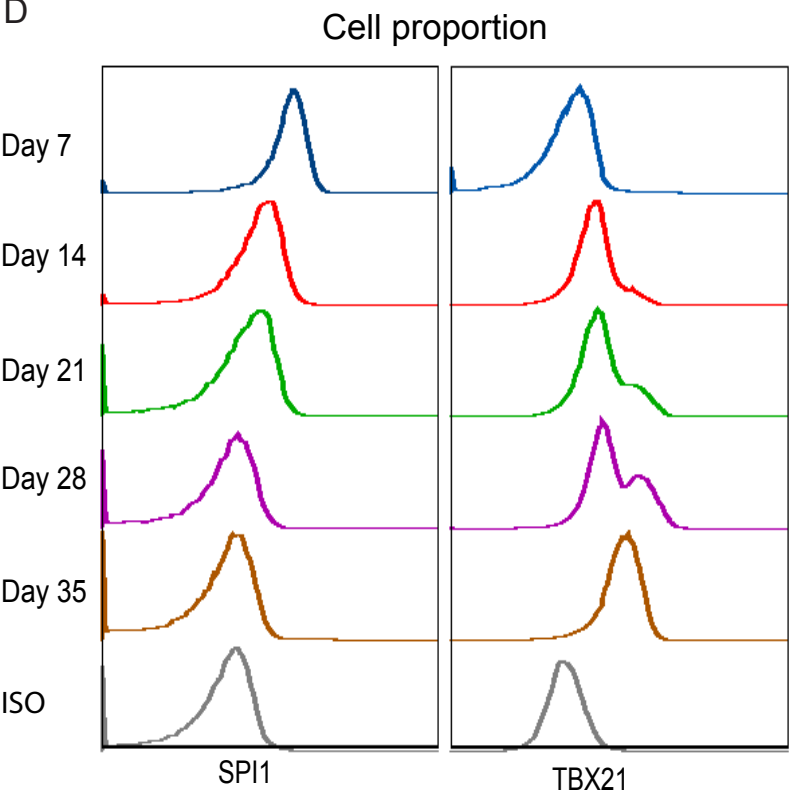

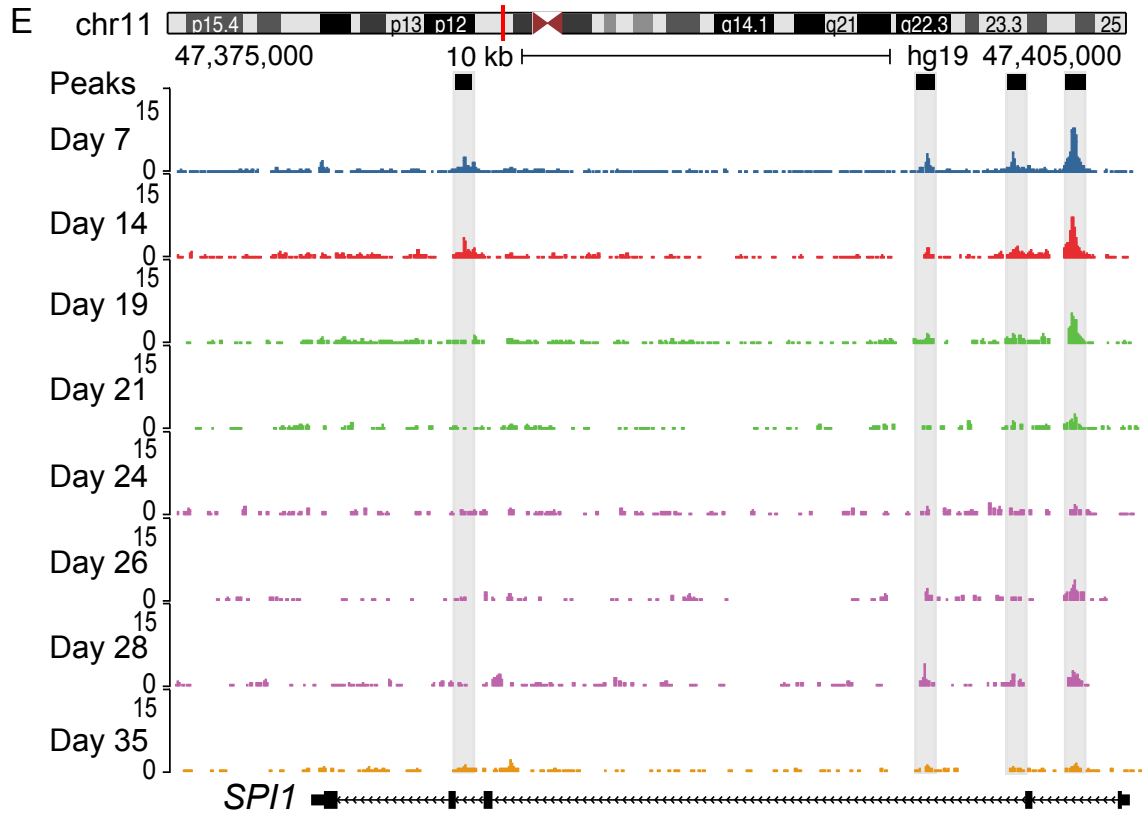
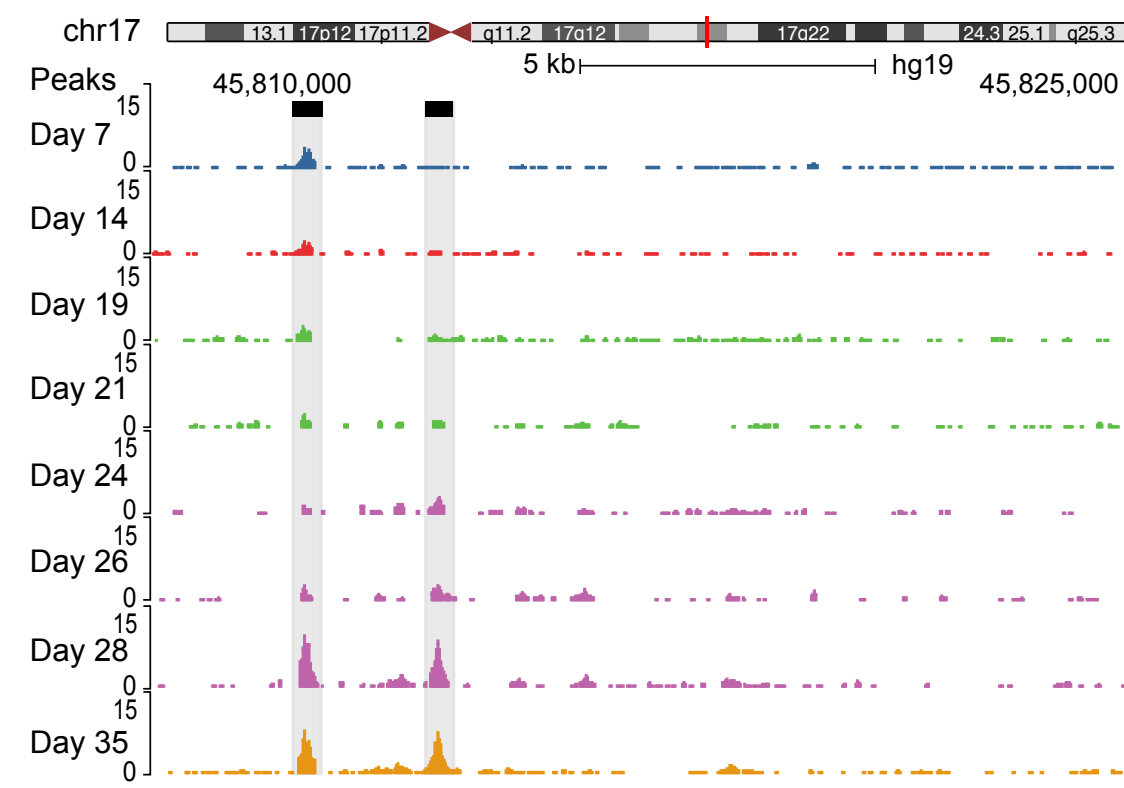

TBX21 


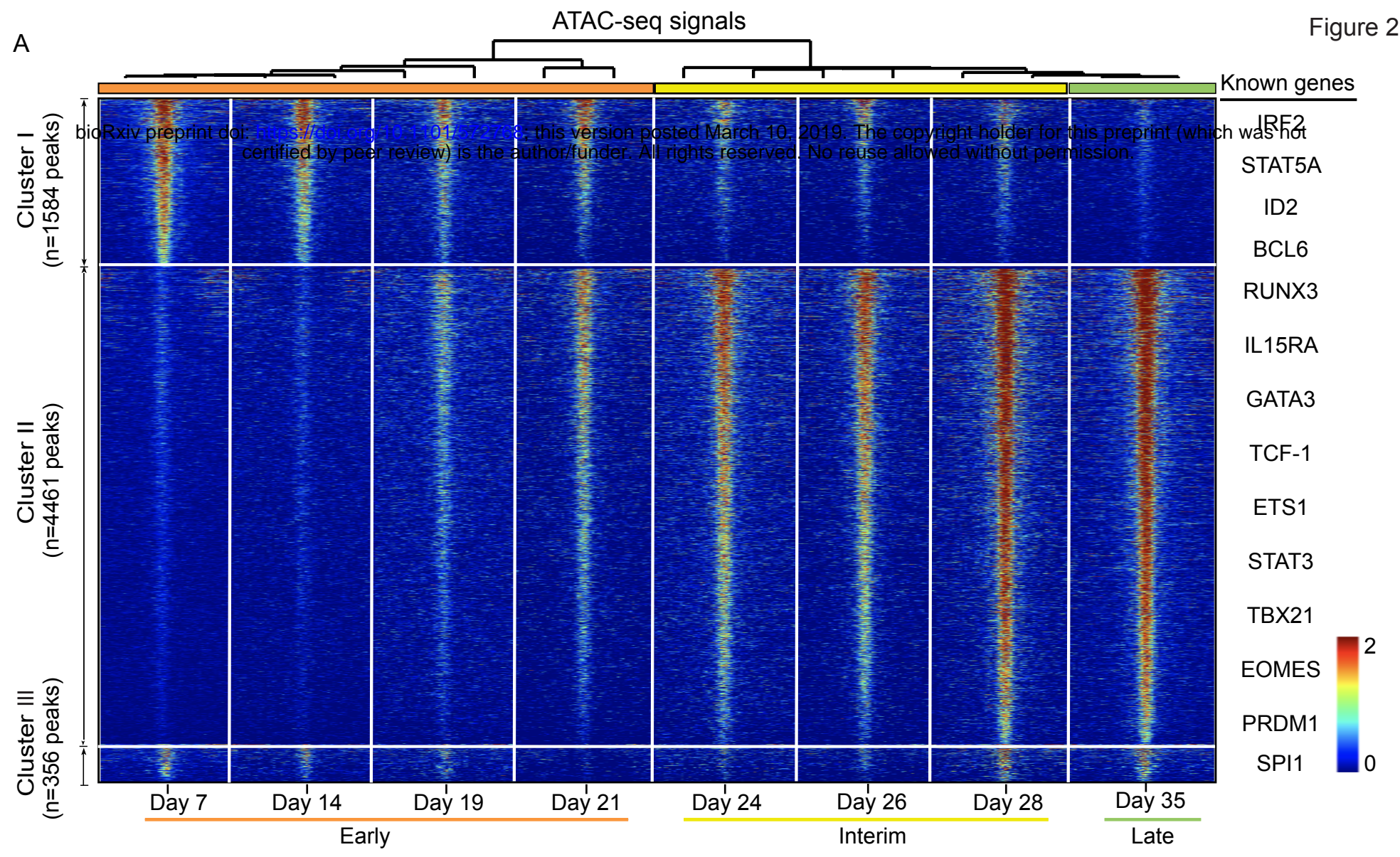

B

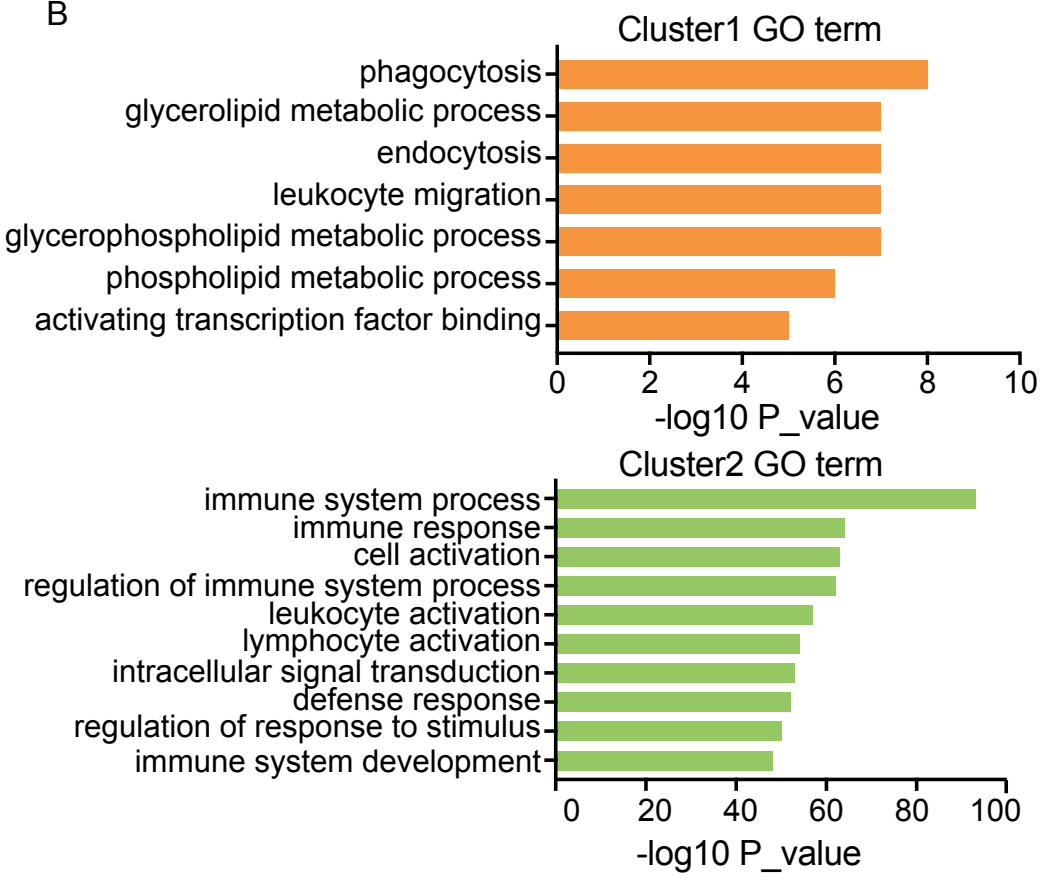

C

Genes more accessible in early of development

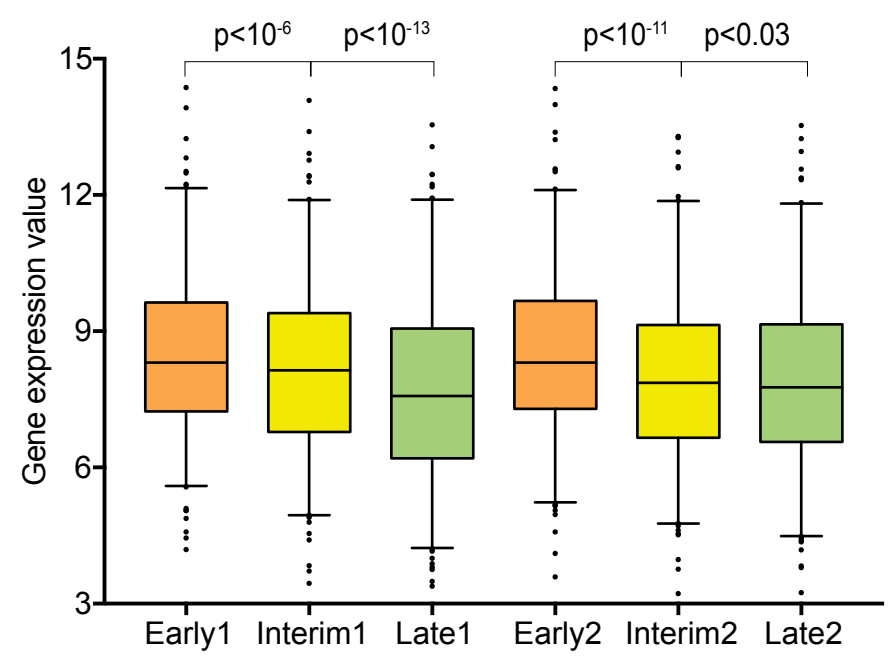

$\mathrm{D}$

- ATAC-seq singals ratio

- Gene expression ratio

- Natural killer cell ratio

glycerophospholipid metabolic process activating transcription factor bindin

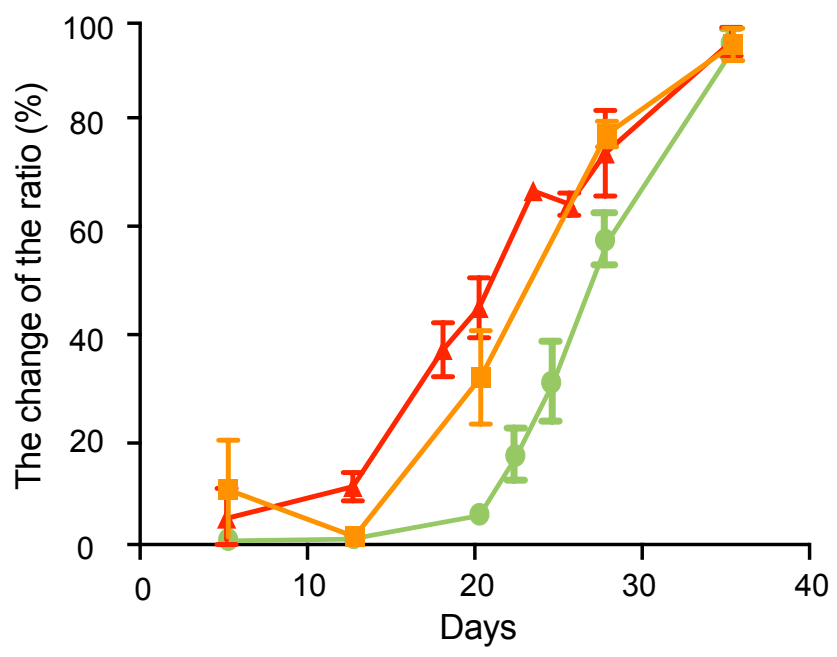

Genes more accessible in late of development

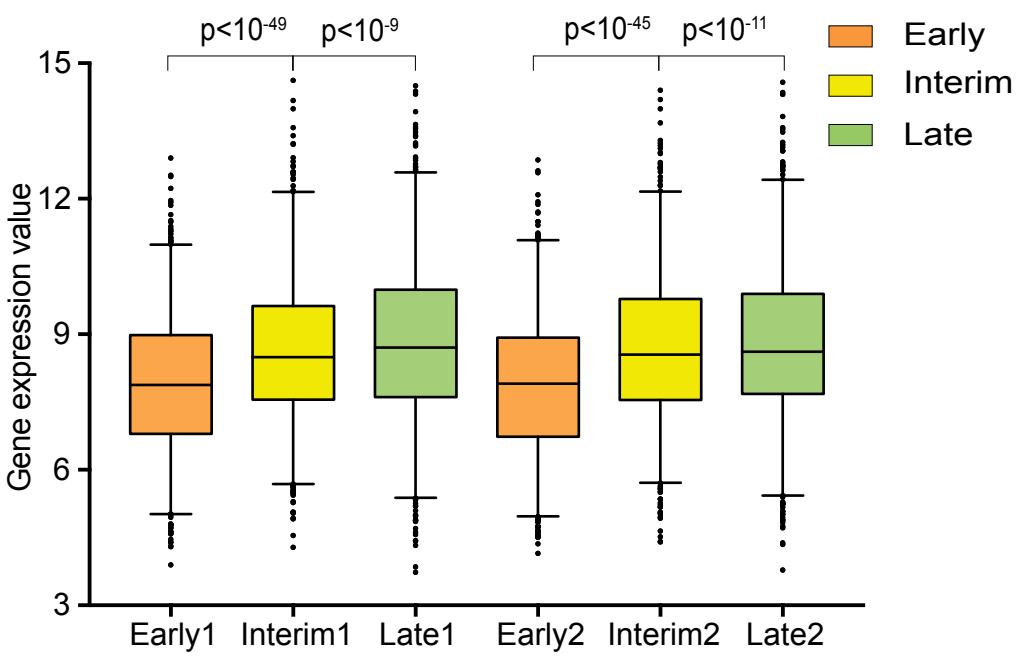


ZNF 416 $\triangle C C G G A A S T$

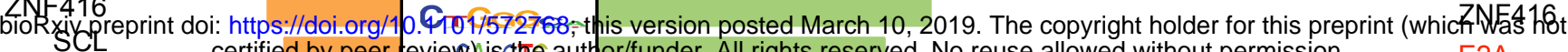

CEBP certified by peer eviev) iscthe auth or/funder. All rights reserved. No reuse allowed without permission.

ERRA

EBF1

$\longrightarrow$ TGA $\subseteq T C A=$

FOSL2

ERRA

STAT6

CAAGGTCA.

EBF1

IRF1

ICCC GGGA

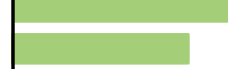

Tbet

STAT5

MEF2C

FOXO1

TFAP2C

B

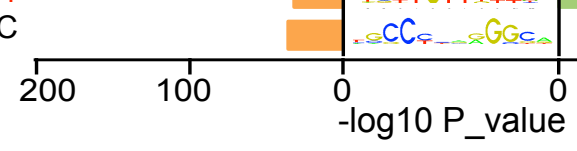
Day 7 21
Day 24 28 Day 35

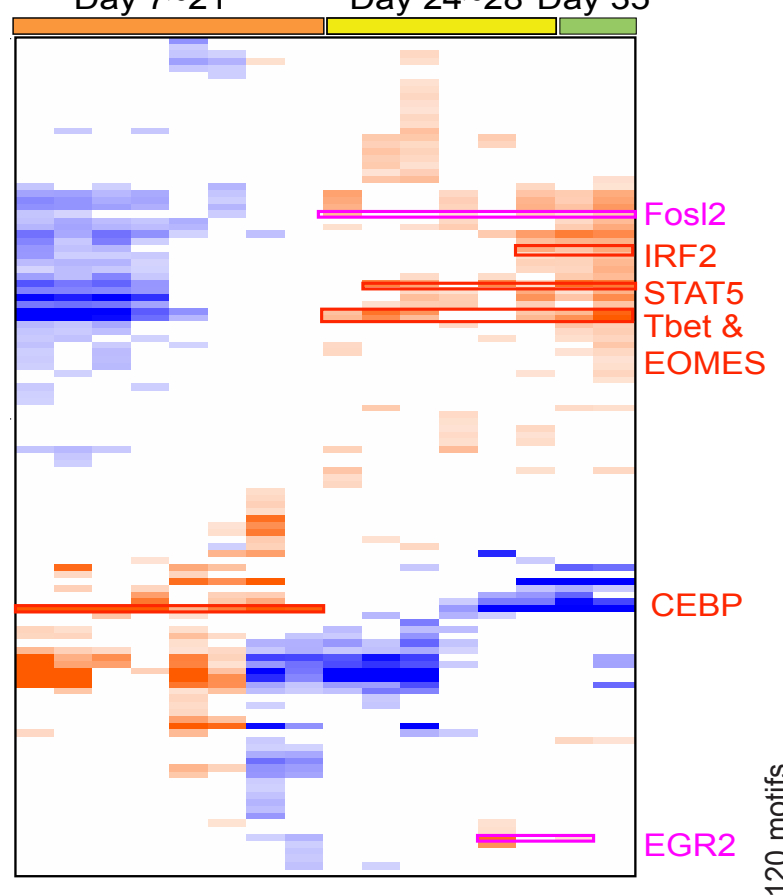

Down 30

log10(Pvalue)
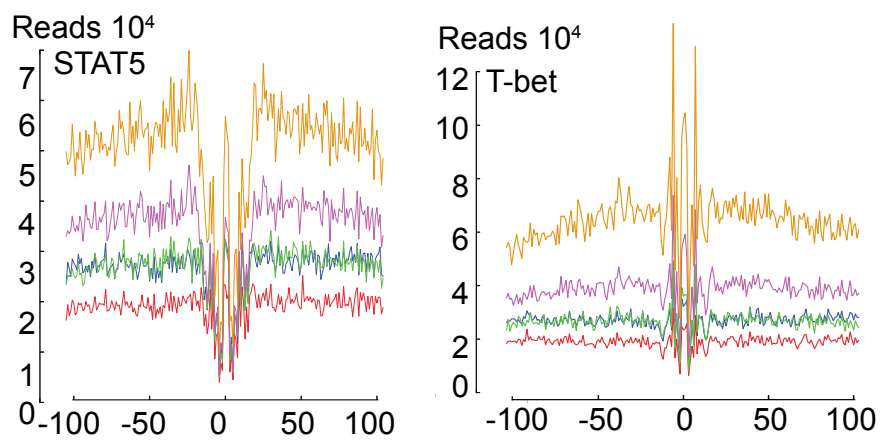

$E$

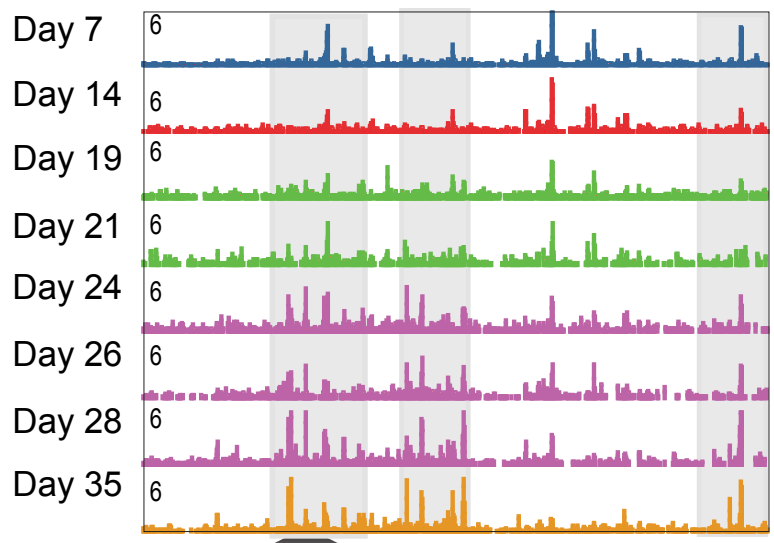

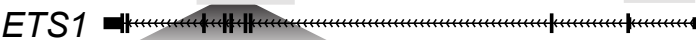

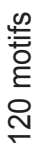

C

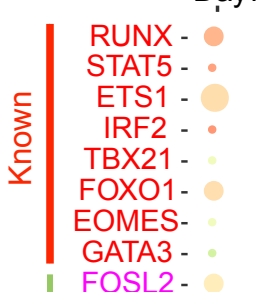

RF2

MEF2C

FOXO1

100

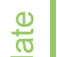

ETV1 -

ATF3 -

$\mathrm{BACH} 2$ -

ARNTL

BIN1 -

NR4A1 -

ERG -

CDC42EP3-

KLF5 -

CEBPB -

ZNF467-

TTK -

MAZ -

ZBTB18-

MEF2C-

ZNF711-

NFE2 -

GFI1B -

SMAD3-

TGIF1 -

RXRA -

MITF -
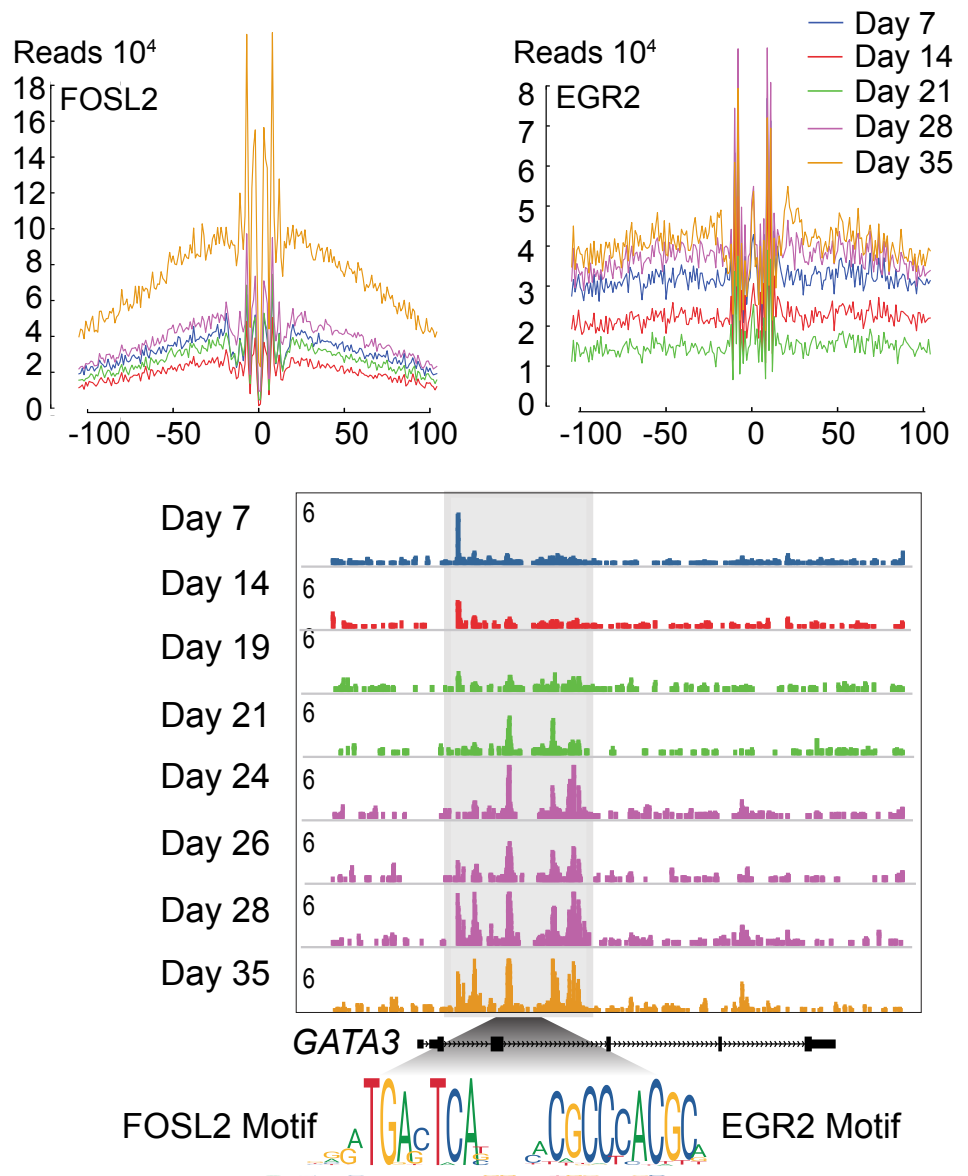

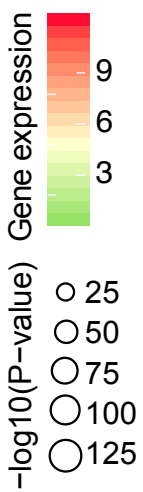

- Day 7

Day 14

Day 28

- Day 35 
A

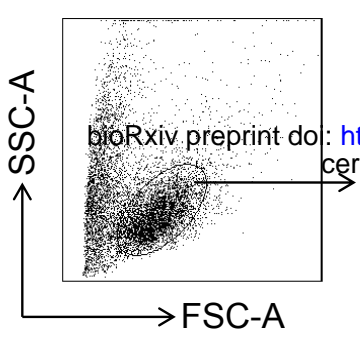

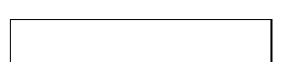

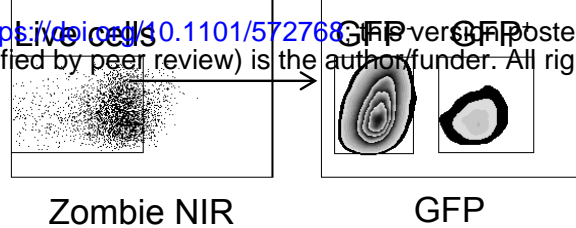

Zombie NIR

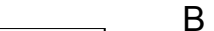

B

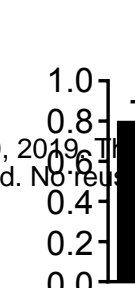

FOSL2

Figure4

EGR2 propint

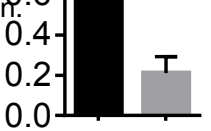

C

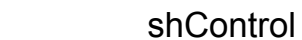

shFOSL2
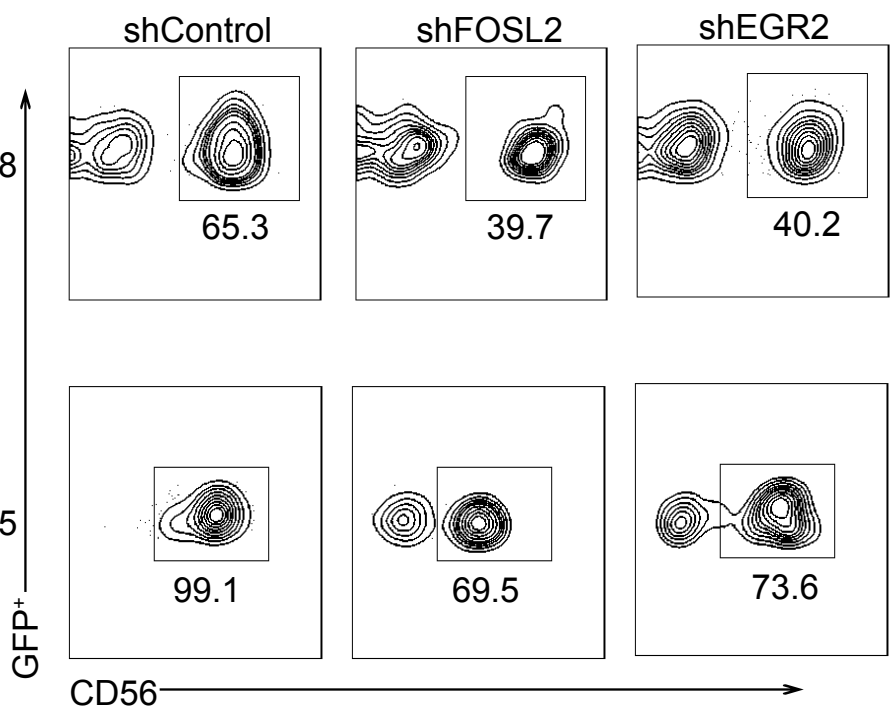

D

GFP $^{+}$Day 28

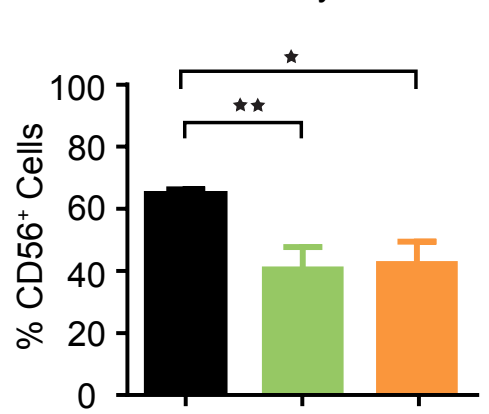

G

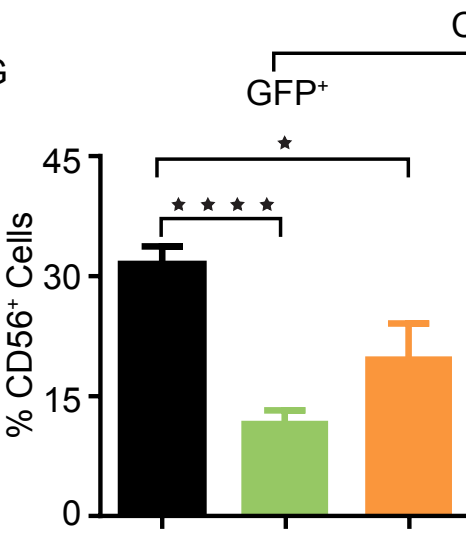

GFP $^{+}$Day 35

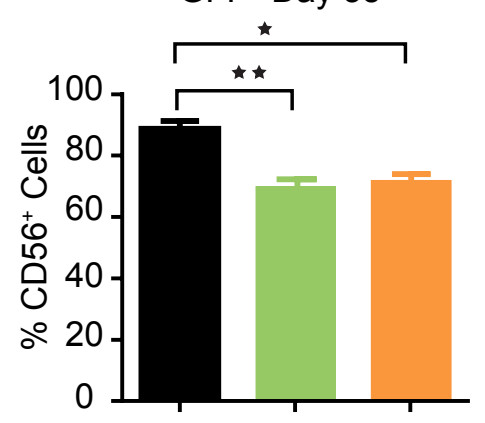

CD11b

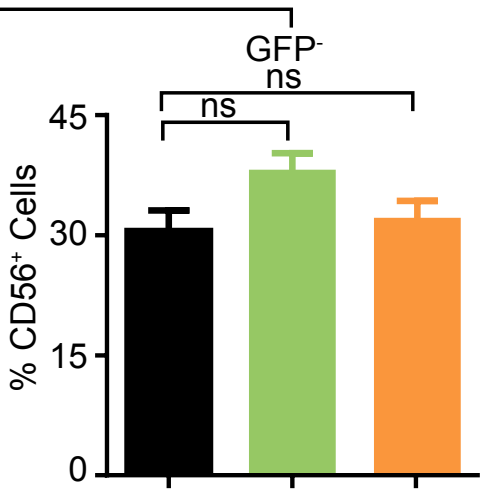

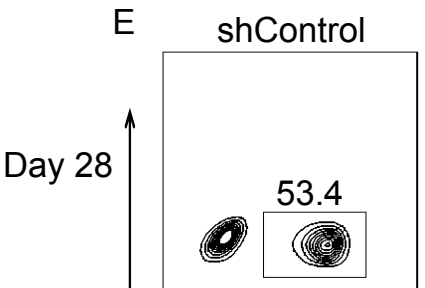

shFOSL2 shFOSL2 0.8- T shEGR2

0.0
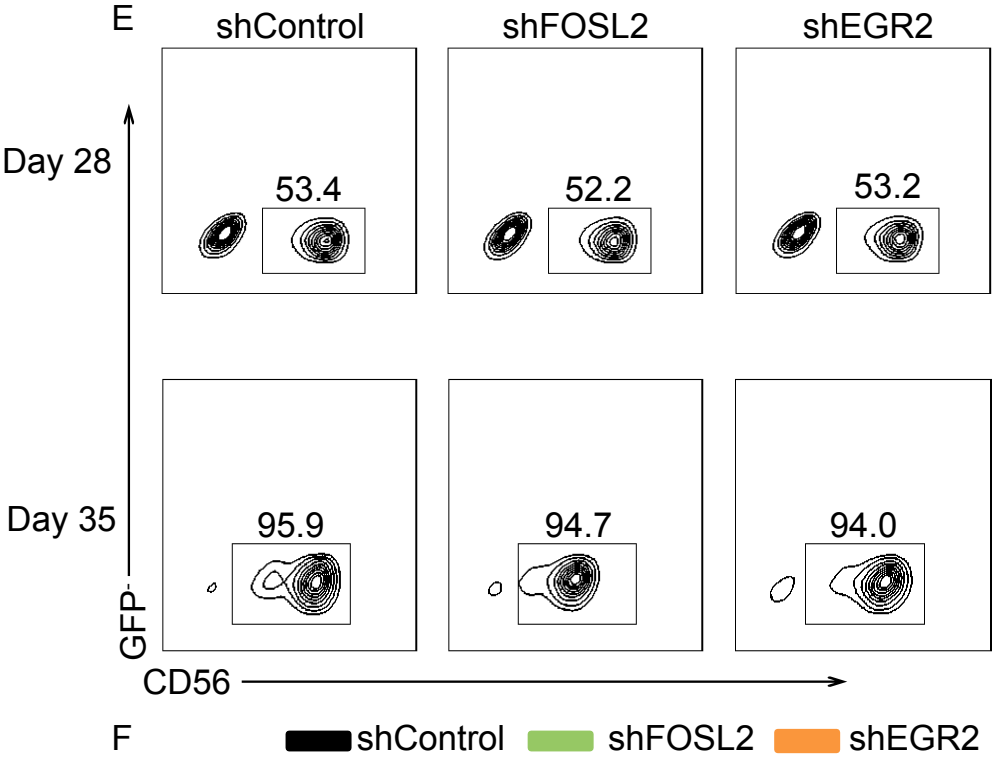

GFP- Day 28
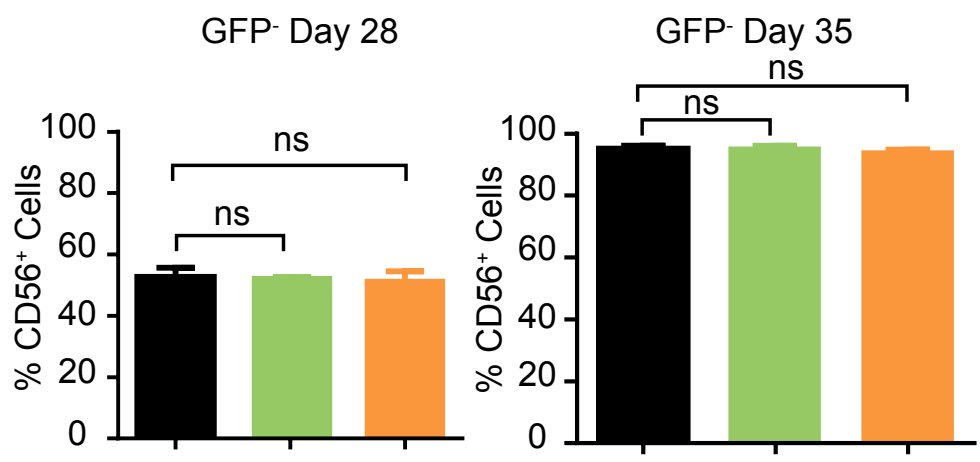

01 
